\title{
Propuesta de innovación docente para la asignatura Pensamiento árabe e islámico clásico (Grado de Estudios Árabes e Islámicos)
}

GRACIA LÓPEZ ANGUITA

Departamento de Filologías Integradas

Área de Estudios Árabes e Islámicos

glopezanguita@us.es

Orcid: https://orcid.org/0000-0003-2635-3149

D.O.I.: http://dx.doi.org/10.12795/JDU.2018.i01.42

Pp.: 751-765

\section{Resumen}

Este texto recoge los resultados de la aplicación del segundo ciclo de mejora docente en la asignatura de Pensamiento Árabo-islámico Islámico Clásico perteneciente al Grado de Estudios Árabes e Islámicos. El Ciclo se centra en el origen de las primeras reflexiones teológicas y, en menor medida, filosóficas, y el ulterior desarrollo de la primera en corrientes definidas. La aplicación del Ciclo de Mejora implica una revisión de contenidos y un cambio de modelo didáctico en el que el alumno construirá progresiva y activamente esos contenidos.

Palabras clave: Experimentación docente universitaria, docencia universitaria, titulación Grado en Estudios Árabes e Islámicos, filosofía islámica, asignatura pensamiento árabo-islámico clásico. 


\section{Contexto}

La asignatura objeto de esta experiencia docente es una optativa de tercer curso. Frente al inconveniente que supone el alto grado de abstracción de los contenidos, cuento con dos ventajas: El reducido número de alumnos (nueve matriculados) y el hecho de que, por estar en tercer curso, los alumnos ya tienen una base previa de Historia del Islam clásico, Corán, Tradición y Derecho islámico. El contenido en el que se ha centrado el segundo Ciclo de Mejora corresponde al tema 4 de la asignatura ("La formación del pensamiento medieval"), es decir, los temas objeto de discusión teológica que propiciaron el nacimiento de esta disciplina, la base coránica de cada uno de ellos y la influencia de la política imperante en cada momento, en este proceso.

Las clases se distribuyen en tres sesiones de ochenta minutos por semana y no comparto la asignatura con ningún otro profesor. El aula posee la infraestructura adecuada para el normal desarrollo de la docencia.

\section{Diseño previo del ciclo de mejora (CMD)}

\section{Mapa de contenidos}

Siguiendo las directrices del profesor y de la bibliografía especializada (PORLÁN, R., 2017), transformé el título del tema en la siguiente pregunta: ¿Cómo se origina y estructura el pensamiento especulativo en el Islam? De igual modo, traté de organizar el contenido en torno a las preguntas del cuestionario que se les entregó antes y después del CMD. Dicho de otro modo, traté de plantear el tema como un enigma al que "iba añadiendo otras cuestiones para que el enigma se mantuviera en el tiempo" (FINKEL, D., 2008). Así, las cuestiones particulares quedan 
insertas en una cuestión general y se puede proceder a "construir socráticamente el conocimiento" y a "razonar antes que memorizar" (BAIN, K., 2007).

Como expreso en el propio esquema, la revisión de contenidos me ha obligado a integrar parte del tema 5 dedicado a filosofía -ya que no puede trazarse una separación tajante en el origen de filosofía y teología- y a dejar fuera una rama de la teología islámica que no era esencial en el conjunto de los contenidos.

Lección 4. La formación del pensamiento medieval

(En mi mapa de contenidos he incluido parte del tema $5^{\circ} \mathrm{y}$ he dejado fuera un subapartado del 4 , atendiendo a la afinidad de contenidos y a la relación lógica entre ellos)

¿CÓMO SE ORIGINA Y ESTRUCTURA EL PENSAMIENTO ESPECULATIVO EN EL ISLAM?

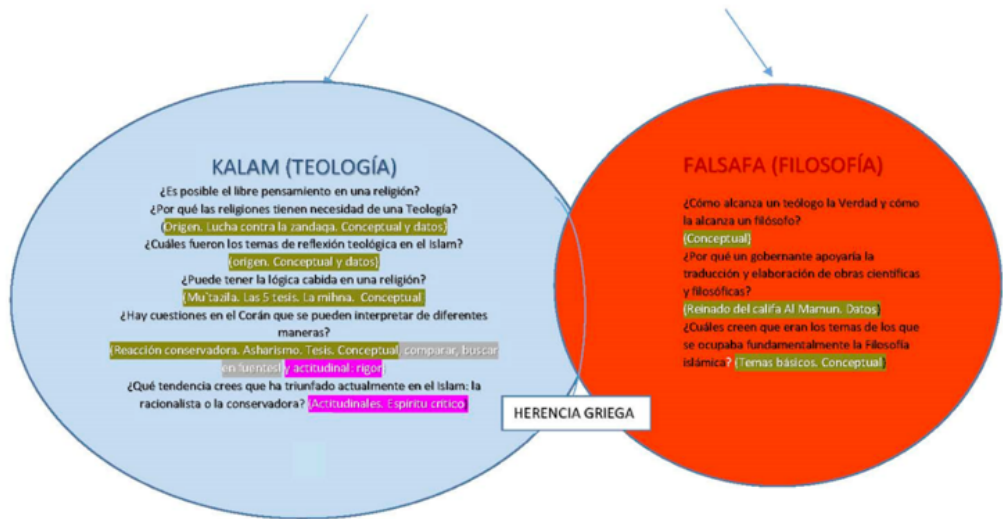

Figura 1 (Para los contenidos teóricos V. CRUZ HERNÁNDEZ, M. 2000 y RAMÓN GUERRERO, R. 1985)

El contenido de la teología es mayor que el de la falsafa, que se trabajará más adelante y que aquí sólo se introduce histórica y temáticamente (de ahí la diferencia de tamaños entre los dos globos). La intersección que he tratado de representar entre ambos diagramas representa la herencia común griega de la que beben tanto la teología como la filosofía.

La clave de colores es la siguiente: verde, contenido conceptual; gris, procedimental y rosa, actitudinal. El contenido procedimental hace referencia a la capacidad de utilizar herramientas de búsqueda de términos en el Corán 
y en otras fuentes, y saber interpretarlas y contrastarlas. Los contenidos actitudinales (además del respeto a los demás alumnos), hacen referencia fundamentalmente al rigor y al espíritu crítico.

\section{Modelo metodológico}

Tras poner en práctica el primer Ciclo de Mejora Docente, diseñé como modelo metodológico posible el mostrado en la figura 2. Mi intención era centrar la clase en el estudiante para que él o ella fuera construyendo o deduciendo el contenido:

Introducción (I.)

Problema (P.)

Hipótesis Alumnos (H.).

\section{Contraste (C.)}

\section{Teoría (T.)}

Figura 2

La introducción suele consistir en un recordatorio de lo visto en la clase anterior y el problema ha consistido en la realización de una tarea que implicaba la contestación a una pregunta. En la práctica terminé unificando contraste y teoría.

Jornadas de Formación e Innovación Docente del Profesorado | № 1 (2018) 


\begin{tabular}{|c|c|c|c|}
\hline $\mathbf{1}$ & \multicolumn{1}{|c|}{ I./P. } & \multicolumn{1}{c|}{ Cuestionario } & $\mathbf{8 0}^{-}$ \\
\hline \multicolumn{2}{|c|}{$\begin{array}{l}\text { Tras una introducción en la que se anuncia la intención didáctica del } \\
\text { cuestionario y se advierte de que no tendrá valor de evaluación, pasamos } \\
\text { a responder el cuestionario inicial individualmente. }\end{array}$} \\
\hline \multicolumn{2}{l}{ Papel y boli. }
\end{tabular}

\begin{tabular}{|c|c|c|c|}
\hline $\mathbf{2}$ & Hip. & Preguntas para resolver colectivamente & $\mathbf{8 0}^{-}$ \\
\hline Recojo y reconduzco en la pizarra las ideas de los alumnos relativas al ori- \\
gen de la teología y la ciencia islámica. Introduzco la denominación téc- \\
nica de los temas que pueden originar controversia en el seno del Islam: \\
Antropomorfización de Dios, Trascendencia de Dios, Libre albedrío, Om- \\
nipotencia divina, Término medio entre la impiedad y la creencia, Corán \\
creado o increado. \\
\hline
\end{tabular}

Tarea para casa: Búsqueda de una serie de aleyas coránicas y situarlas dentro de los apartados conceptuales arriba indicados (Antropomorfización, etc.)

\begin{tabular}{|c|c|c|c|}
\hline 3 & Hip. / Contr. & Puesta en común de la tarea & $80^{\circ}$ \\
\hline \multicolumn{4}{|c|}{ Discusión y corrección de la tarea. } \\
\hline \multicolumn{4}{|c|}{$\begin{array}{l}\text { Papel y boli. } \\
\text { Recurso on-line de búsqueda coránica. } \\
\text { Proyector }\end{array}$} \\
\hline
\end{tabular}

\begin{tabular}{|l|c|c|c|}
\hline $\mathbf{4}$ & \multicolumn{1}{|c|}{ T. } & \multicolumn{1}{c|}{ Exposición teórica. } & $\mathbf{6 0}^{-}$ \\
\hline \multicolumn{2}{|c|}{ Exposición teórica que sintetiza y amplía lo visto antriormente. } \\
Powerpoint sencillo en el que se exponen el Kalām, la Mu`tazila, la \\
zandaqa. \\
Aquí introduzco por primera vez nombres propios y fechas. \\
\hline $\begin{array}{l}\text { Powerpoint. } \\
\text { Proyector. }\end{array}$ \\
\hline
\end{tabular}

5

P.

Lectura en clase.

$20^{-}$

Jornadas de Formación e Innovación Docente del Profesorado I № 1 (2018) Esta obra se distribuye con la licencia Creative Commons Reconocimiento-NoComercial-SinObraDerivada $\quad 4.0$ Internacional (CC BY-NC-ND 4.0.) 
Para ver un ejemplo real de zandaqa (libre pensamiento/herejía), leemos en voz alta y comentamos un texto de un autor perteneciente a esa corriente.

Fotocopias.

Tarea para casa: Lectura de capítulo del libro de Abed Al-Yabri, El legado filosófico árabe. Alfarabi, Avicena, Avempace, Averroes, Abenjaldún. Lecturas contemporáneas.

\begin{tabular}{|c|c|c|c|}
\hline $\mathbf{6}$ & $\mathbf{T .}$ & Exposición teórica. & $\mathbf{8 0}^{-}$ \\
\hline \multicolumn{2}{|l|}{ Exposición teórica para explicar la reacción conservadora: el aš arismo } \\
\hline Pizarra
\end{tabular}

\section{Cuestionario}

Plantear cuestiones de teología y filosofía islámica a través de preguntas que resultaran familiares o cotidianas -evitando terminología abstrusa- que fueran abiertas y que implicaran, en ocasiones, una valoración personal, (v. PORLÁN, R. p. 78) ha supuesto un verdadero reto para mí y, al mismo tiempo y a la vista de los resultados obtenidos, ha sido una fuente de satisfacción y motivación como docente. Por otra parte, este tipo de contenido es extraordinariamente propicio para generar discusión, debate y crítica.

A continuación, expongo el cuestionario con el tipo de respuesta organizado de menos madura y peor argumentada (A) a más madura y mejor argumentada (C), basándome en las respuestas de los alumnos.

1. ¿Crees que es posible el libre pensamiento en el seno de una religión "que se está formando"?

a) El libre pensamiento es incompatible con la religión.

b) Es posible hasta cierto punto.

c) Sí, porque en ese estadio la religión aún no está afianzada y permite su exploración/Es necesario para formarla. 
2. ¿Puede tener la lógica cabida en una religión -en este caso, la islámica-?

a) No, en las religiones hay ciertas ideas estipuladas y son ilógicas. En el Islam más aún que en otras.

b) Sí. (En los ejemplos dados el alumno no ha entendido bien "lógica")

c) Sí con contradicciones / Sí pero unida al dogma.

3. ¿Por qué las religiones tienen "necesidad" de una teología?

a) El alumno no tiene claro lo que es la teología.

b) Por la necesidad de explicar qué es Dios, etc.

c) Defensa y adaptación.

4. ¿La teología contribuyó a que se desarrollaran otras ciencias?

Todos han respondido afirmativa y correctamente. Los tipos de ciencias se han distribuido así:

Por un lado, hermenéutica, gramática y ciencias puramente "religiosas" y, por otro, las que hoy entendemos más como ciencias (matemáticas, química, etc.). Ambas tipologías me parecen correctas y no sabría cómo clasificarlas.

5. ¿Cuáles crees que fueron los primeros temas de reflexión teológica en el Islam?
a) Vida de Muhammad.
b) Legislación.
c) Unicidad de Dios.

6. ¿Hay cuestiones en el Corán que puedan interpretarse de diferente manera; se te ocurre alguna?

a) Respuesta mal argumentada. Confusión entre conceptos. Todo el Corán es interpretable. Ningún ejemplo concreto.

b) Ejemplos concretos correctos.

7. ¿Qué tendencia crees que ha triunfado en la teología islámica al cabo de los siglos: la racionalista o la conservadora?
a) Racionalista
b) Las dos
c) La conservadora / Depende del país 
8. ¿Por qué un gobernante apoyaría y financiaría la traducción de obras científicas y filosóficas?

a) Para comprender a las culturas circundantes.

b) Mejorar la cultura propia. Prosperidad.

c) Intereses políticos y económicos. Propaganda. El verdadero sentido de la empresa traductológica y filosófica de los primeros califas era luchar desde el plano intelectual contra los elementos no islámicos que habian tenido el poder en lo que había sido el imperio persa. Nadie ha respondido con esta respuesta porque es parte del contenido de la asignatura. Si el CMD funciona según lo previsto, debería encontrar esa respuesta en el cuestionario posterior.

9. ¿Cómo alcanza un teólogo la "verdad" y cómo la alcanza un filósofo?

a) Rezando / respuestas confusas.

b) Por medio de la razón y por medio de la Fe y la reflexión sobre el texto revelado.

10. ¿Cuáles crees que eran los temas de los que se ocupaba fundamentalmente la filosofía islámica clásica?

a) Confusión de términos, conceptos y del sentido de la pregunta.

b) Separar la lógica de la teología. Temas coránicos.

c) Ética, política, metafísica.

\section{Aplicación del ciclo de mejora}

Recojo a continuación, a modo de diario de clase, cómo se desarrolló en la práctica, el segundo ciclo de mejora docente.

\section{Sesión 1}

La distribución del cuestionario previo causa un revuelo inesperado: constato el miedo a "todo lo que parezca un examen" que tienen los alumnos. Muchas de las preguntas las dejan en blanco no por ignorancia, sino por, según me cuentan ellos mismos, miedo al juicio que yo 
haga sobre ellos. Muchas de las dudas que surgen acerca de preguntas concretas del cuestionario me sirven para empezar a testar y aclarar cosas en la siguiente clase.

\section{Sesión 2}

Por recomendación del profesor del Curso de innovación docente, vuelvo a pasar el cuestionario pues el hecho de que bastantes preguntas hayan quedado en blanco, invalida parte del proceso del segundo Ciclo de Mejora. Los convenzo de que no puntúa este primer cuestionario y de que son ellos los principales beneficiados con esta estrategia. En esta ocasión los resultados son mejores.

Continúo con el plan previsto para la segunda sesión pero ya con un retraso en la planificación temporal debido a este incidente del cuestionario.

Recojo, reconduzco, añado ideas que los alumnos me van dando en un diálogo oral entre ellos y yo sobre la pizarra. El contenido son los temas que originaron debate en el comienzo de la teología islámica. El resultado es bastante bueno con participación activa de todos los alumnos.

(Tarea para casa, procedimental y de contenido: búsqueda de aleyas coránicas y distribución de las mismas en los siguentes temas: Antropomorfización de Dios, Trascendencia de Dios, Libre albedrío, Omnipotencia divina, Término medio entre la impiedad y la creencia, Corán creado o increado. Estos términos han sido explicado en la tarea anterior en la pizarra).

\section{Sesión 3}

Faltan algunos alumnos y vienen otros que no habian estado en la clase anterior. Este hecho unido a la dificultad en la búsqueda de aleyas a través de la herramienta online, hace que me vea obligada a distribuirlos en grupos de alumnos heterogéneos (españoles, extranjeros, musulmanes, etc.), repartir los versículos y hacer y corregir la tarea de casa en clase.

Los alumnos van asimilando y familiarizándose a través de esta tarea con conceptos y terminología pero vamos con un día de retraso con respecto al diseño previo. 


\section{Sesión 4}

Exposición de contenido organizado ya en corrientes, fechas y nombres propios con powerpoint. Buena acogida por parte de los alumnos que han organizado y afianzado un tipo de contenido que es muy abstracto.

Tanto en el diseño previo como en la puesta en práctica de este segundo ciclo, he tenido que recurrir a la "clase magistral" porque aún me veo insegura con el modelo Problema->Hipót. alumnos->Contraste.

\section{Sesión 5}

Termino la exposición que había comenzado la clase anterior (arrastro retraso de tiempo desde la segunda sesión). Leemos en clase un fragmento de un autor considerado como perteneciente a la zandaqa (herejía) para ver un ejemplo real de la producción intelectual de esta corriente de pensamiento.

Presentación teórica de la corriente ortodoxa de pensamiento en la pizarra con menos tiempo del previsto. Es la última parte de contenido teórico del segundo Ciclo de Mejora. La sensación es que los alumnos van siguiendo y comprendiendo el contenido. A través de las dudas o cuestiones que se debaten veo que tienen presentes algunas de las preguntas del cuestionario inicial y tratan de relacionar el contenido con ellas.

Entre la quinta y la última sesión tengo una estancia en el extranjero. Les mando como tarea la lectura de un fragmento de un filósofo árabe contemporáneo que hace una interpretación crítica de la teología y la filosofía islámica para discutirlo en clase a mi regreso.

\section{Sesión 6}

A mi vuelta sólo un estudiante había leído el texto. Los demás, según me dicen, o habían olvidado la tarea o no sabían que había que hacerla. Con la participación del estudiante que sí lo había leído, hacemos una síntesis del texto.

Una vez más, me doy cuenta de la importancia de pedirles los ejercicios por escrito. Al no haber hecho la tarea 
final que es fundamental para cerrar el ciclo y responder a algunas de las preguntas del cuestionario, traslado al día siguiente tanto la discusión sobre el capítulo como el cuestionario final.

\section{Sesión 7 (extra)}

Ya con los resúmenes puestos por escrito, tanto la clase como el cuestionario final resultan muy satisfactorios, como expondré en el epígrafe siguiente.

\section{Evaluación del aprendizaje}

Fueron seis los alumnos que respondieron al cuestionario previo y posterior al Ciclo de Mejora y en función de esos cuestionarios elaboré las escaleras de aprendizaje (v. PORLÁN, R., 2017, pp. 82-83). A partir de ellas resumo a continuación y de manera lineal los resultados. El número de la izquierda indica la respuesta previa y el de la derecha, la respuesta posterior. Recordemos que la respuesta A corresponde a un menor grado de acierto y complejidad y la C (o B, en los casos en que no había necesidad de mayor división en peldaños de conocimiento), al mayor y deseable:
1) $A: 3 / 1 B: 1 / 2 C: 2 / 3$
2) $A: 2 / 0 B: 3 / 0 C: 1 / 6$
3) $A: 1 / 1 B: 2 / 1 C: 3 / 4$
4) $A: 6 / 1 B: 0 / 5$

A la pregunta 4 del cuestionario previo y posterior, los estudiantes respondieron afirmativamente. Marco la diferencia entre el peldaño $A$ y el $B$ en virtud de la idoneidad de los ejemplos puestos y de la argumentación que, en prácticamente en todos los casos, supera la inicial.

5) $A: 1 / 0 \quad B: 2 / 2 \quad C: 3 / 4$

A pesar de que el número de estudiantes que respondieron Cantes y después del ciclo de mejora está muy cerca, la calidad y precisión de las respuestas del cuestionario a 
posteriori supera con creces al previo. Se constata ya un manejo de conceptos teológicos.
6) $A: 4 / 3 B: 2 / 3$
7) $A: 1 / 0 \quad B: 1 / 3 \quad C: 4 / 3$

Se comprueba en esta pregunta un gran progreso en el nivel de argumentación y en el empleo de los nombres de las corrientes teológicas islámicas.
8) $A: 1 / 0 \quad B: 4 / 3 \quad C: 1 / 3$
9) $A: 2 / 1 B: 4 / 5$
10) $A: 3 / 0 B: 1 / 3 C: 2 / 3$

Como se desprende del contraste entre las respuestas pre y post, la evolución de la práctica totalidad de los alumnos es favorable. Algo extensible a todos, es que manejan nombres propios de personas, corrientes y conceptos que antes no manejaban, y que emplean más y mejores argumentos. Junto al uso riguroso de terminología, aplican casi de manera sistemática una visión crítica en sus respuestas. La escalera de aprendizaje me ha sido especialmente útil para detectar qué ideas no han calado aún en el alumno y los prejuicios que éste tiene. En este sentido, y creo que no es una cuestión baladí, he podido comprobar con satisfacción cómo algunas de las ideas preconcebidas que sobre el Islam tienen algunos alumnos, son superadas tras la aplicación del Ciclo de Mejora.

\section{Conclusiones}

En general, la aplicación de este CMD, a pesar de que en ocasiones me he visto obligada a retomar la clase magistral, me ha resultado extremadamente positivo. El nivel de compromiso por parte del estudiante y de trabajo por parte del docente es mayor, pero los resultados, sin duda, lo merecen. He tomado conciencia de las ideas erróneas de las que parto al dar por supuestos los modelos mentales del alumno y el contenido que ya manejan y de lo 
motivante que puede llegar a ser para ellos trasladarles la resolución de determinados problemas. He sido consciente también de la importancia de planificar el tiempo dedicado a cada actividad, de pedir cualquier actividad por escrito y de calificar numéricamente esos ejercicios para motivar al alumno y proporcionarle una guía de su progreso en la asignatura. La distinción entre contenidos actitudinales, procedimentales y teóricos (con sus subdivisiones) para mi ha sido revelador, porque hasta ahora sólo calificaba los teóricos y a veces, de manera vaga, los actitudinales.

El hecho de tener que plasmar en un esquema los contenidos, la tipología y las relaciones entre ellos me ha ayudado a organizar la docencia y las actividades de manera óptima. Es más, la elaboración del esquema me sugería actividades relacionadas con el contenido.

He advertido que los alumnos, a pesar de la reticencia inicial con algunas actividades concretas, se van acostumbrando a la nueva dinámica, por lo que resulta interesante plantearla para toda la asignatura. Por ejemplo, cada vez que mando una lectura, ellos ya me envían por escrito un resumen sin que yo se lo pida.

Con respecto al cuestionario, me he dado cuenta al terminar el segundo ciclo de que no debe ser tan largo y de que tengo que "atreverme" a hacer preguntas incluso más cotidianas y desafiantes. Aparte del cambio global de modelo metodológico de docencia, hay ideas muy concretas con las que me quedo para su futura aplicación, como son la importancia de poner por escrito todo, el diario del profesor (que había llevado en alguna asignatura anterior pero siempre orientándolo a si el alumno trae o no la tarea hecha), la idea de traer las preguntas teóricas al ámbito de la experiencia cotidiana del alumno, la evaluación no sólo de los contenidos teóricos, la calificación con una nota numérica concreta cada ejercicio, así como el uso de herramientas on-line que antes desconocía. 
Estoy convencida de que este tipo de contenido teológico-filosófico no habría sido tan satisfactoriamente asimilado por ellos, ni habría sido tan ameno, si se hubiese hecho a través del modelo tradicional de clases magistrales. 


\section{Bibliografía}

Bain, K. (2007), Lo que hacen los mejores profesores de universidad, Valencia: Universitat de Valencia.

Cruz, M. (2000), Historia del pensamiento en el mundo islámico, Madrid: Alianza.

Finkel, D. (2008), Dar clașe con la boca cerrada. Valencia: Universitat de Valencia.

Porlán, R. (2017), Enseñanza universitaria. Cómo mejorarla. Madrid: Morata.

Ramón, R. (1985), El pensamiento filosófico árabe. Madrid: Cincel.

Yabri, M. A. (2016), El legado filosófico árabe. Alfarabi, Avicena, Avempace, Averroes, Abenjaldún. Lecturas contemporáneas, Madrid: Trotta.

Jornadas de Formación e Innovación Docente del Profesorado | № 1 (2018) Esta obra se distribuye con la licencia Creative Commons 